\title{
COMPETENCIA COMUNICATIVA Y NUEVAS FORMULAS DOCENTES: LA EMERGENCIA DE NUEVOS MODELOS DE COMUNICACIÓN DIDÁCTICA
}

José Manuel Cardoso-Belo1: Universidade de Trás-os-Montes e Alto Douro. Portugal jbelo@utad.pt

\section{RESUMEN}

A fin de delinear una propuesta teórica el artículo presenta a una síntesis de las investigaciones realizadas sobre la interacción didáctica, la organizando de acuerdo a tres perspectivas: proceso-producto, la sociolingüística y etnografía, y el análisis del discurso. A continuación, se aborda el concepto de competencia comunicativa. Por último, se proponen dos modelos a través de representación gráfica: uno en la competencia comunicativa y otro en la comunicación didáctica. Al tratar con la dinámica de su interacción en las relaciones, nuestro enfoque se centra en la relación entre los individuos así como en los propios individuos. Suponemos, por tanto, una perspectiva interactiva y pragmática. Nuestra posición es que la competencia es algo que se crea en el contexto de una relación $\mathrm{y}$, en este sentido, se define la competencia comunicativa. El modelo relacional de la competencia comunicativa didáctica que se presenta es un modelo integral, basado en los principios teóricos de la relación que busca integrar los componentes claves de la comunicación con un proceso dinámico. Sin embargo, debido a que el modelo incorpora una gran cantidad de variables, se pone un reto con respecto a los medios para medir la competencia en el ámbito de la relación.

PALABRAS CLAVE: Comunicación didáctica - Competencia comunicativa - Modelo Teoría.

\footnotetext{
${ }^{1}$ Autor correspondiente

José Manuel Cardoso Belo: Universidade de Trás-os-Montes e Alto Douro - Portugal

Correo: jbelo@utad.pt
} 


\title{
COMMUNICATIVE COMPETENCE AND NEW TEACHING FORMULAS: THE EMERGENCE OF NEW MODELS IN CLASSROOM COMMUNICATION
}

\begin{abstract}
In order to delineate a theoretical proposal, the paper presents a synthesis of research on teaching interaction organized according three perspectives: process-product, sociolinguistics and ethnography, and discourse analysis. If follows the concept of communicative competence. Finally, we propose two models: one on communicative competence and the other on didactics or classroom communicative competence. In dealing with the dynamics of their interaction in relationships, our approach focuses on the relationship between individuals as well as individuals themselves. We assume, therefore, a pragmatic and interactive perspective. Our position is that competence is something that is created in the context of a relationship and, in this sense, communicative competence may be defined. The relational model of didactics or classroom communicative competence presented is an integral model, based on the theoretical principles of the relationship, that seeks to integrate the key components of communication with a dynamic process. However, the fact that the model incorporates a large number of variables poses a challenge as for the means to measure the competence within the relationship.
\end{abstract}

KEY WORDS: Classroom Communication - Communicative competence - Model Theory.

\section{INTRODUCCIÓN}

Vivimos en una época de profundos cambios tecnológicos, sociales, políticos, económicos y, por supuesto, estos cambios tienen un impacto en los sistemas de educación, y, más concretamente, en las aulas y los procesos de comunicación que se producen allí.

La sociedad de la información en que vivimos ha contribuido claramente a los cambios observables en la escuela y en la relación entre maestros o profesores y estudiantes. Una estricta disciplina tradicional fue seguida por una liberalización que muchos consideran excesiva; el déficit crónico de información fue seguido de una explosión y masificación de la información; local, regional, nacional, continental y global son (pueden ser) instantáneos y simultáneos. A lo largo de la historia, el cambio ha sido permanente, pero nunca como hoy que fue tan rápido y drástico. 
Durante mucho tiempo, la Escuela casi monopolizó el acceso à la información (saber). Sin embargo, la imagen actual de cambios rápidos y continuos, donde la posibilidad de acceder y obtener información en tiempo real y en muchos lugares es un hecho, será interesante investigar los dispositivos desarrollados por la Escuela y, por extensión, por los profesores, para adaptarse a esta 'nueva' situación que cambia rápidamente.

La revolución de los medios de comunicación implica un cambio en las condiciones ambientales y no tiene sólo un carácter aditivo o lineal. De hecho, lo que nos enfrentamos ahora es completamente nuevo y más complejo; la sobrecarga de información no es simplemente un exceso, apunta a otra dimensión que requiere otras soluciones, exige un nuevo conjunto de técnicas, lo que significa la necesidad de nuevos patrones de percepción, de comprensión y de evaluación, es decir, un nuevo tipo de educación y de comunicación educativa.

Dado el enorme flujo de información se requiere, entonces, nuevas actitudes y habilidades, ya que no es suficiente, como en el esquema cultural en vigor hasta unos años, saber leer correctamente para manejar la información disponible. El desarrollo de habilidades comunicativas son, por lo tanto, uno de los intentos de dotar a las personas con las habilidades necesarias para hacer frente a la "sociedad de la información".

En este escenario, que hace el profesor? que estrategias para adaptarse al cambio usa? que medios utiliza? que recursos tiene? cómo comunica en un entorno saturado de información? No es nuestra intención, en el espacio disponible, ofrecer respuestas definitivas a todas las preguntas anteriores, pero tan sólo, indicar algunas de las posibilidades de adaptación a las exigencias derivadas de la "nueva" situación de la educación.

De hecho, en principio, el profesor enseña comunicando, es decir, informa, explica, mostra, demostra, etc.; a menudo hace recurso a la exposición, continúa, no obstante, con un discurso en el que las perífrasis, metáforas y paráfrasis, por ejemplo, son presencias constantes. Es decir, la herramienta de comunicación más utilizada en el aula por profesores y alumnos es, todavía, el lenguaje en sus formas verbal y noverbal.

Cabe destacar que la adquisición de una competencia de comunicación sólo es posible a través de la comunicación y, por lo tanto, los procesos de transmisión y de aprendizaje son también, y sobre todo, procesos de comunicación. Así, la relación entre comunicación y enseñanza es doble: por un lado, la enseñanza es un proceso comunicativo y, en segundo lugar, una de las funciones de la enseñanza es permitir la adquisición de una competencia comunicativa. 
Esto significa que las condiciones de la comunicación humana son al mismo tiempo las condiciones de la enseñanza, y por lo tanto, la enseñanza es fundamentalmente condicionada por reglas de comunicación.

Es con esta imagen de fondo que desarrollamos las siguientes consideraciones que culminarán con la presentación de propuestas de dos modelos: uno en la competencia comunicativa y, otro, en la competencia de comunicación didáctica.

\section{DESARROLLO}

\subsection{Comunicación Didáctica y Teorías de la Comunicación}

Aunque el estudio de la Comunicación como tal, es decir, con la designación de Comunicación, en la Universidad es relativamente reciente, el número de publicaciones sobre este tema ha aumentado de manera exponencial.

La palabra comunicación es dificil de definir. El nombre es abstracto y, al igual que muchas otras palabras, tiene muchos significados. En 1976, Dance y Larson han dado cuenta de la existencia de ciento veintiséis diferentes definiciones de comunicación. Hoy, el número de opciones disponibles es incomparablemente superior, por el simple hecho de que el número de publicaciones sobre el fenómeno es en la actualidad mucho mayor de lo que era hace unos 35 años.

Lo que se dijo acerca de la comunicación puede, de alguna manera, volver en relación con la Didáctica. De hecho, la cantidad de estudios sobre la didáctica, en su versión general, así como sobre las didácticas específicas, también es impresionante y sólo nos referimos a la denominación generalmente utilizada en países de Europa continental y sus zonas de influencia. Por otra parte, como es sabido, la tradición de investigación en Didáctica es muy anterior a la de la Comunicación stricto senso. Entonces, ¿cómo caracterizar la Comunicación Didáctica?

Se puede decir que esta es una disciplina teórico-práctica que, desde una perspectiva multidisciplinar, busca estudiar el origen, naturaleza y funciones de la comunicación humana con fines didácticos, con la intención de definir y dar nueva forma, a través de un enfoque crítico y de renovación, cómo poner los fundamentos de la enseñanza y de la práctica en el área del lenguaje, verbal y no-verbal, y de la comunicación. Abarca, por tanto, el tratamiento de elementos axiales en los procesos educativo y comunicacional. Se centra, por un lado, en la redefinición tanto del perfil del docente como del fundamento epistémico de su acción tecnodidáctica y, en segundo lugar, en la identificación de necesidades y requerimientos de actualización en la comunicación interpersonal. 
La disciplina pretende ser una interfaz entre los conocimientos científicos existentes y la redefinición de los contenidos educativos, y propone, basada en el reconocimiento y tratamiento de los princípios y contenidos de la lingüística, la teoría de la comunicación y las disciplinas en competencia, un modelo de acción didáctica centrado en el estudio, análisis y producción de lenguajes verbales, paraverbales y icónicos, preferentemente dentro de la comunicación real y social en entorno educativo institucional.

Al revisar la literatura sobre las teorías de la comunicación en la que se analizaron más de tres docenas de teorías, y teniendo en cuenta que la comunicación es una amplia zona de la actividad humana, hemos retenido las características o aspectos que, en nuestra opinión, están relacionados com la comunicación didáctica. Hemos dejado fuera todos los aspectos o teorías sobre la comunicación intrapersonal y de masas, reteniendo sólo aquellas características que también puedam ser consideradas en relación com la comunicación interpesonal.

Los múltiples aspectos y dimensiones de las teorías de la comunicación desafian una clasificación clara. No existe un sistema de categorías que se adapte perfectamente a organizar este material, aunque diversos esquemas pueden utilizarse. Así que tratamos de utilizar las propuestas de Griffin (Griffin, 1991), Littlejohn (Littlejohn, 1996), y Craig (Craig, 1999) para apoyar a nuestro análisis.

A pesar de la importancia que tienen ambas tipologías para la comprensión de la comunicación como proceso esencialmente humano, hemos seleccionado sólo a aquellas que parecen más relevantes para nuestros propósitos.

\subsection{En Busca de una Propuesta Teórica}

És con este fondo que presentaremos nuestra contribución a la creación de una teoría de la comunicación didáctica. Pero, antes de hacerlo, y para un mejor ajuste, nos gustaría presentar un panorama general de la investigación realizada en la interacción didáctica² ${ }^{2}$.

A pesar de que hemos identificado, recopilado y estudiado escritos sobre el análisis de la interacción publicado durante los últimos cuarenta años, hemos limitado nuestra lista a los textos que, en nuestra opinión, son más relevantes.

Teniendo en cuenta nuestros objectivos, hemos organizddo la investigación sobre la

\footnotetext{
2 En este ambito, la investigación se há llevado a cabo principalmente en los EE.UU. donde presenta una dinâmica extraordinária, y en los países anglosajones. En Europa continental no parece haber una gran tradición de investigación en este campo. En Portugal, el estudio que consideramos más relevante sobre la interacción en el aula es el de Emília Ribeiro Pedro (1982), O Discurso na Aula:
} 
interacción en el aula desde tres perspectivas: la del proceso-producto, la sociolinguística y etnográfica, y la análisis del discurso. Esta organización, por sí misma, no necesariamente refleja un acuerdo académico generalizado sobre la manera de organizar los resultados de la investigación ni agota todos los estudios realizados.

El término "proceso-producto" se refiere a la relación central, o interacción, entre los procesos de enseñanza y los productos de los estudiantes. En uno de los textos fundacionales de esta perspectiva, Flanders (Flanders, 1970) proporciona un conjunto de ejemplos para apoyar la afirmación de que el clima en el aula se puede medir de manera objectiva y fiable, y que este clima se relaciona con la eficacia docente. Una parte sustancial de su testimonio se basó en el sistema de observación com su nombre para registrar el comportamiento verbal del profesor. Sin embargo, desde su creación hs habido muchas reservas sobre su efectividad y Delamont y Hamilton (Delamont \& Hamilton, 1976), entre otros, criticaron este tipo, norte-americano, dominante de análisis de la interacción en el aula y propusieron la adición de otros métodos antropológicos. Emihovich (Emihovich, 1989) subrayó el hecho de que los estudios etnográficos se caracterizan por su atención al uso del lenguaje, al contexto social más amplio, y a la realidad común de la vida cotidiana en las aulas.

En 1979, Mehan dijo que, debido a que las interacciones en el aula tienen características de conversación específicas, que las distinguen de las interacciones sociales cotidianas, requieren un estudio y atención especiales. Cochran-Smith (Cochran-Smith, 1984) y Bryce-Heath (Bryce-Heath, 1983) han llamado la atención sobre el hecho de que el aprendizaje ocurre tanto dentro como fuera del aula a través de una socialización gradual, más do que a través de una enseñanza directa y, en consecuencia, es necesario estudiar las interacciones sociales de los estudiantes fuera de la escuela para intentar comprender sus interacciones en el aula. Por otro lado, Au y Mason (Au \& Mason, 1983), por ejemplo, han analizado las formas en que las estructuras de participación facilitan o impiden interacciones educacionales positivas.

Algunos sociolingüístas apoyan la idea de que se debe estudiar la lengua-en-uso como resultado de determinados contextos sociales. Por ejemplo, Cazden, John y Hymes (Cazden, John \& Hymes, 1972) han utilizado un enfoque sociolingüístico para explorar las discontinuidades entre los lenguajes de las culturas caseras de los niños y las demandas comunicativas de las culturas escolares. 
Un estudio de Gumperz y Cook- Gumperz (Gumperz \& Cook- Gumperz, 1982) reveló que el origen social de un estudiante puede indicar una forma de adquisición y práctica del discurso que entra en conflicto com las opiniones del profesor, lo que puede producir percepciones erróneas acerca de las prácticas de los estudiantes que pueden influir en su evaluación; y Yamauchi y Tharp (1995) dicen que, para participar plenamente en la vida del aula, los estudiantes tienen que adquirir más de una mera competencia lingüística; tienem que asimilar las reglas sociolingüísticas que acompañan y embasan los estilos discursivos de la cultura associada con el lenguaje.

El trabajo de Edwards y Mercer (Edwards \& Mercer, 1987) señala que las escuelas tienen sus propias normas o cultura epistemológica para identificar y demostrar conocimiento $\mathrm{y}$, por lo tanto, las interacciones educacionales se deben basar en el conocimiento compartido que debe ser construído a través de actividades y discursos compartidos.

El estudio de Mehan (1985) se refiere al concepto de la reflexividad del lenguaje, es decir, mientras que los participantes construyen de forma interactiva el tejido social de la clase a través del discurso, este discurso también afecta a su participación en el aula.

Con el fin de comprender la naturaleza de la relación entre las oportunidades para aprender, las prácticas sociales y discursivas y sus consecuencias para los estudiantes, la investigación de Crawford, Castanheira, Dixon y Green (Crawford, Castanheira, Dixon \& Green 2001) plantea la cuestión central en cualquier estudio de una aula: ¿quién puede decir o hacer qué y con quién, cuándo y dónde, en qué circunstancias, en relación a qué acciones o artefactos, para qué propósitos, y con qué resultados?

Por lo tanto, la perspectiva sociolingüística, con su atención a las estructuras de participación, permite a los investigadores examinar en detalle las formas en que los miembros de la aula, los profesores y los estudiantes, constroyen sus formas de ser y de relacionarse a través del uso de la lengua en contextos particulares. De hecho, los sistemas educativos contemporâneos se enfrentan a un creciente número de situaciones en las que los hablantes gestionan diferentes variedades del habla que reflejan sistemas gramaticales y culturales "diferentes pero iguales", o, en otras palabras, mientras que las lenguas y variedades del mismo idioma son diferentes, en última instancia son iguales en valor comunicativo y potencial retórico.

\subsection{La interacción en el aula como proceso sociolingüístico}

Entre muchos aspectos, el tema resumidamente referido arriba sugiere que los problemas de comunicación en el contexto escolar pueden no ser debidos a problemas de lenguaje, si al uso del contexto y la interpretación hecha por los involucrados en la comunicación (profesor y estudiantes). 
Ciertos estudios sobre las prácticas en el aula han demostrado que algunas de las características del sistema de comunicación didáctica dán o negan el acceso al aprendizaje en situaciones en las que el mensaje realmente verbalizado era en realidad una parte del sistema total.

Estos estudios nos alertan sobre la naturaleza comunicativa del sistema social del aula y el acceso a oportunidades de aprendizaje ser socio-comunicativamente determinado y al hecho de que el aprendizaje no es una simple cuestión de flujo de información en la que las palabras del docente son recojidas por los estudiantes sino más bien un proceso interactivo que depende de la capacidad de los profesores y estudiantes para crear participación en la conversación, es decir, los estudiantes y profesores deben trabajar para obtener la atención de los demás y la capacidad de lo realizar está condicionada por la ecología social de la clase.

Para tratar de entender mejor el papel del lenguaje en el aprendizaje, algunos estudios han sugerido que será más importante tener en cuenta el discurso que a la gramática y que la naturaleza del discurso escolar difiere bastante con relación al experimentado fuera de la escuela, no como el reflejo de los diferentes valores culturales o focando ciertas rutinas de enseñanza, pero, en esencia, constituyen la tarea de la escuela.

El estudio de Mehan (Mehan, 1985) es un buen ejemplo de cómo la investigación sociolinguística ha demostrado que lo que se tiene que aprender es a menudo secundario respecto a cómo se presenta la información, lo que conduce a la esperanza de que el cambio de estilos de enseñanza y de aprendizaje hace más accesibles los contenidos de los conocimientos escolares, si la alfabetización lingüística, si la matemática, o otra. Una mejor comprensión entre el estudiante y docente sobre los estándares y código(s) sociolinguístico(s) de cada uno dellos debería proporcionar una transmisión más directa del conocimiento como contenido de lo que debe ser aprendido.

En el marco de la investigación sociolingüística, el enfoque a los procesos de la escuela se centra en la interacción entre supuestos lingüísticos, contextuales, y sociales que interactúan para crear las condiciones para el aprendizaje en el aula y que se aplicán a interpretaciones hechas en el contexto de los eventos discursivos que caen dentro de la interacción diaria. Las características de determinadas situaciones en el aula se ven como algo que ocurre con regularidad, rutinas discursivas unidas a través de las prácticas cotidianas de los profesores y estudiantes, es decir, existen características de estas rutinas que son similares en todos los contextos del aula y otras que varían según la progresión de la enseñanza. 
Sin embargo, el interés en los eventos discursivos también tiene como objetivo explorar las estructuras de participación que rigen el tipo y la cantidad de interacción que componen el evento y cómo las expectativas de los participantes sobre estas estructuras entran en la interpretación de mensajes particulares, basándose, pues, en una tradición académica diferente, los traballos sobre pragmática lingüística y la teoría de los actos de habla (Austin, 1962);( Searle, 1970); (Grice, 1975); (Levinson, 1983).

La conversación de cualquier tipo requiere la cooperación activa entre los productores de información y oyentes que proporcionen retroacción, ya sea por respuestas directas o a través de formas alternativas de signos indirectos. Dicha cooperación no puede darse por sentada porque, para asegurar la cooperación de conversación, los posibles hablantes deben llevar a otros a cooperar, es decir, deben servir al menos alguna información temprana sobre el resultado deseado.

Gumperz (Gumperz, 1982) señala que cualquier tipo de interpretación en situaciones de enseñanza en el aula, por lo general, visto como actividade centrada en tareas orientadas a objetivos, depende del uso de señalización o de estrategias de contextualización por los participantes con el fin de establecer un entorno propicio para la comunicación eficaz. Para los miembros de la clase, el movimiento diario a través del tiempo, de evento en evento, es parte esencial del conocimiento comunicativo acerca de cuando ocurre un evento, como se producen cambios en las actividades, como un cambio se convierte en un nuevo contexto que indica qué esperar y cómo interpretar lo que se dice.

Así, la interacción en el aula se debe a un proceso de inferencia conversacional basado en la producción y la percepción de los participantes de las señales verbales y no verbales que contextualizan la actividad del habla. Al contextualizar estas propuestas, los participantes reconocen las actividades como parte de secuencias de habla más amplias a través de las cuales identifican los contextos.

Así, los participantes crean y emplean esquemas para enmarcar las interpretaciones situadas de cada uno de ellos $y$, juntas, las contextualizaciones forman un sistema que crea una cadena de significados a través de la cual la interacción progresa. Aunque estos fenómenos conversacionales tengan interpretaciones situadas y localizadas, también deben proporcionar un hilo conductor a través del cual los participantes construyen com el tiempo cadenas específicas de comprensión. Para entender mejor lo que el enfoque interactivo a la comunicación implica para la manera en que vemos los datos lingüísticos y lo que los intercambios conversacionales nos dicen acerca de la interacción entre los supuestos lingüísticos, socioculturales, y contextuales, debemos reconsiderar a un concepto clave - la competencia comunicativa. Desde nuestro punto de vista, cualquier propuesta de modelo o teoría de la comunicación didáctica es incompleta sin la consideración de este concepto. 


\subsection{La competencia comunicativa}

La creación de un concepto de competencia comunicativa debe mucho a los lingüistas Chomsky (Chomsky, 1965) y Hymes (Hymes, 1972a,b), y su historia aún se puede remontar a la Grecia clásica, donde la elocuencia y la oratória eran cuestiones fundamentales de la educación - los cinco cânones de la retórica de Aristóteles son quizá el primer modelo de comunicación competente. La competencia comunicativa ha sido un tema central en la investigación sobre comunicación interpersonal y intercultural y ha recibido cierta atención en el campo educativo.

Una tendencia en la investigación sobre la competencia que ha estado tratando de comprender la importancia de los factores contextuales es el punto de vista relacional, cuyo énfasis está puesto en los procesos dinámicos, relacionales, de la comunicación.

Aunque el contexto y los supuestos socioculturales han comenzado a surgir en algunos modelos explicativos, las ideas acerca de lo que se rige por normas respecto al habla y como el significado se transmite siguen basándose en gran medida en conceptos originarios del análisis gramatical basado en la frase.

Hablamos de la lengua y la cultura, la lengua y el contexto, como si fueran entidades separadas, fuera del mensaje y que pueden ser comparadas a objetos concretos, limitados.

Los estudios de la competencia comunicativa tienen que tratar con signos lingüísticos a um nivel de generalidad que trasciende los límites del sistema gramatical y concentrarse en los aspectos relacionados con el significado o interpretación más general que el contenido de la frase. Por otra parte, está claro que lo que tenemos que procesar en los intercambios conversacionales es diferente de lo que se aplica en la decodificación de frases aisladas.

Cabe señalar que las estrategias verbales para negociar interpretaciones conversacionales son en su mayoría indirectas. La información no se expresa abiertamente en el contenido superficial, sino que debe ser inferida sobre la base de supuestos tácitos obtenidos a través de las experiencias previas de interacción. Los mecanismos de señalización indirecta difieren de los signos lexicalizados en la medida en que, tal como las señales no-verbales, son inherentemente ambiguos. Cualquier declaración simple está siempre sujeta a múltiples interpretaciones y las personas deciden la interpretación aceptable analizando lo que Austin ha designado como 'uptake', es decir, el proceso de conversación a través del cual las lineas de razonamiento se desarrollan o modifican. 
Recientes estudios empíricos indican que los malentendidos atribuibles a diferencias sistemáticas sin ser detectadas en la señalización de convenciones se producen con una frecuencia considerable. Estas convenciones se crean a través de la experiencia interactiva prolongada en familia, entre amigos, y redes de relaciones. Una vez establecidas a través de la práctica, vienen a servir como recursos comunicativos que canalizan las inferencias a lo largo de determinadas líneas y el conocimiento de su funcionamiento se convierte en una condición previa para la participación activa en los encuentros verbales. Este conocimiento es de un tipo que no puede ser fácilmente adquirido a través de la lectura o la educación formal en el aula, porque el contacto personal es necesario en situaciones que permitan maximizar la retroacción.

Un número significativo de fallos o interrupciones en la comunicación puede ser debido a inferencias basadas en diferencias no detectadas en las estrategias de contextualización. Por lo tanto, ¿cómo documentar el funcionamiento de las estrategias de contextualización? El problema es complejo y requiere un análisis más detallado de lo que puede presentarse aquí, pero la explicación más probable residirá en las convenciones discursivas para la señalización de la co-referencialidad.

\subsection{Una Propuesta de Modelos}

Teniendo en cuenta lo que se ha dicho hasta ahora, parece legítimo intentar proponer un modelo de competencia comunicativa y otro en relación a la comunicación didáctica, cuyas formas gráficas se presentan a continuación y que vamos a comentar. El Gráfico 1 presenta un modelo de competencia comunicativa desarrollado en Wiemann (Wiemann, 1977) y Wiemann y Kelly (Wiemann \& Kelly, 1981), complementado com el análisis de las propuestas de Canale y Swain (Canale \& Swain, 1980), Cupach y Imahori (Cupach \& Imahori 1993), Gumperz (Gumperz, 1981 1984), Hymes (Hymes, 1972b), Imahori y Lanígan (Imahori \& Lanígan, 1989), Savignon (1983), Spitzberg y Hecht (Spitzberg \& Hecht, 1984), van Ek (van Ek, 1975), y Wiemann y Bradac (Wiemann \& Bradac, 1989). 


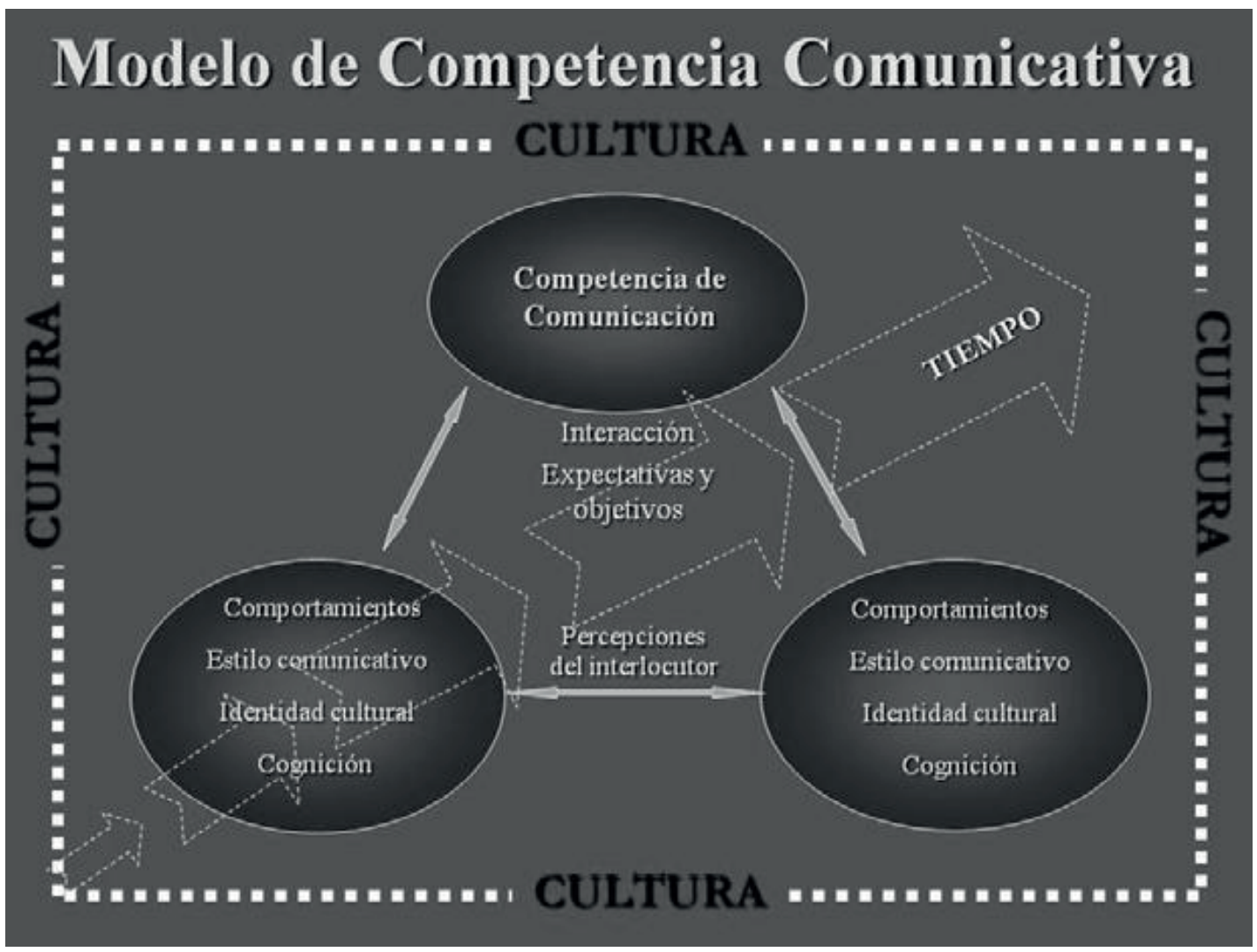

Figura 1. Modelo de competencia comunicativa

Fuente: elaboración propia

Como ya se mencionó, uno de los objetivos de este trabajo es contribuir al desarrollo de un modelo de competencia de comunicación didáctica basado en una perspectiva sistémica teórica, relacional, que integre teorías de la comunicación interpersonal que ya existen, especialmente las basadas en la sociolingüística.

\section{CONCLUSIONES}

Al analizar los distintos modelos de la competencia comunicativa, se puede señalar que, en general, cada tipo de modelo hace hincapié en una sola dimensión de la competencia. Por lo tanto, teniendo en cuenta las limitaciones de cada tipo de modelo, nosotros vemos como deseable un modelo multidimensional de la competencia comunicativa.

La mayoría de los modelos de competencia de comunicación se refiere a la competencia a nivel individual y la considera cómo un concepto que reside en el individuo. Otros modelos se centran en el sistema relacional, si bien no explican la variación individual o cultural. Buscamos que nuestro enfoque se centre en la relación entre los individuos, así cómo en los propios individuos, ocupándose de la dinámica de su interacción en las relaciones. 
Asumimos, por tanto, una perspectiva interactiva y pragmática. Nuestra posición es que la competencia es algo que se crea dentro de una relación. En este sentido, adaptando y complementando Gumperz (Gumperz, 1981, 1984), nosotros definimos la competencia comunicativa como el conocimiento de las convenciones lingüísticas y comunicativas que los hablantes deben tener para iniciar y mantener una relación conversacional satisfactoria para ambas partes mediante la creación de mensajes adecuados y efectivos, siendo que la participación o envolvimiento conversacional es una condición previa necesaria a la comprensión.

Hace tiempo que psicólogos interculturales consideran la comunicación entre 'culturas' en el sentido más amplio del término, que abarca, por ejemplo, el género, la ocupación, área geográfica, la capacidad o discapacidad física, etc. (Collier, 2000). En este sentido de la palabra, cada individuo representa una mezcla única de pertenencia a culturas étnicas o nacionales sino también a un número casi infinito de lo que se designan como "pequeñas culturas": familia, grupo de edad, grupo profesional, grupo político, redes sociales, etc. (Holliday, 1999).

Por lo tanto, este modelo relacional tiene como objetivo prevenir complicaciones culturales teniendo en cuenta la cultura y su componente contextual, es un modelo 'culturalista' y difiere de los modelos relacionales ya existentes cuando trata de centrarse en el proceso de comunicación dentro de la relación y de los individuos en el ámbito de una situación. Su resultado puede ser definido cómo satisfacción relacional, es decir, la realización de los objetivos alcanzada en el marco interpersonal facilitador y de colaboración provoca sentimientos de satisfacción en la relación y su continuidad se desea. Sin embargo, las relaciones incompetentes pueden inducir sentimientos de desagrado y la inadecuada distribución de control y/o orientación para lo(s) objetivo(s) puede dar lugar a la insatisfacción.

Debido a su naturaleza relacional, este modelo tiene pocas restricciones sobre el número de miembros en el ámbito de una relación competente. No presupone que la relación es diádica y permite relaciones de cualquier dimensión, siempre que permitan la existencia de los elementos de la competencia.

La naturaleza teórica del modelo se sitúa en el punto de vista de la teoría de sistemas, en el tratamiento de la competencia comunicativa como algo que resulta de un sistema interactivo: la relación. En la línea de Watzlawick, Beavin y Jackson (Watzlawick, Beavin \& Jackson 2000), este modelo propone que los componentes de la competencia relacional sean vistos como interrelacionados para formar un todo, permitiendo que la competencia sea vista desde una perspectiva holística. Así, el modelo debe incluir no sólo los individuos en el ámbito de una relación, sino también los factores contextuales.

Los procesos relacionales que se producen en el marco de este modelo se pueden explicar por la teoría de la acomodación de la comunicación (Giles et al., 1987): en 
El modelo relacional de competencia de comunicación didáctica presentado en el Gráfico 2 es un modelo integral, basado en los principios teóricos de la relación y que busca integrar componentes importantes de la comunicación como un proceso dinámico.

Nussbaum (Nussbaum, 1992), y Baringer y McCroskey (Baringer \& McCroskey, 2000) han señalado que la investigación existente implica a menudo que una sola persona el profesor - influye en la otra persona - el estudiante. Es decir, muchas investigaciones tienen una visión unidireccional de los procesos de comunicación en el aula. En este marco, Baringer y McCroskey (Baringer \& McCroskey, 2000, p. 179) han concluído que "ambos los campos, de la comunicación y de la educación, han prácticamente ignorado la interacción circular entre estudiante y profesor en el aula”.

A su vez, Nussbaum (Nussbaum, 1992, p.176) también señaló que el énfasis actual sobre el comportamiento del profesor refleja un modelo de comunicación "proceso-producto" que "ha producido investigación estática y lineal," que es "muy evidente en la investigación sobre educación y comunicación que ignora los efectos que tienen los estudiantes sobre el comportamiento de los profesores". Del mismo modo, Waldeck et al. (Waldeck et al., 2001) han llegado a la conclusión de que deberíamos prestar más atención a la interacción mutua entre profesores y estudiantes.

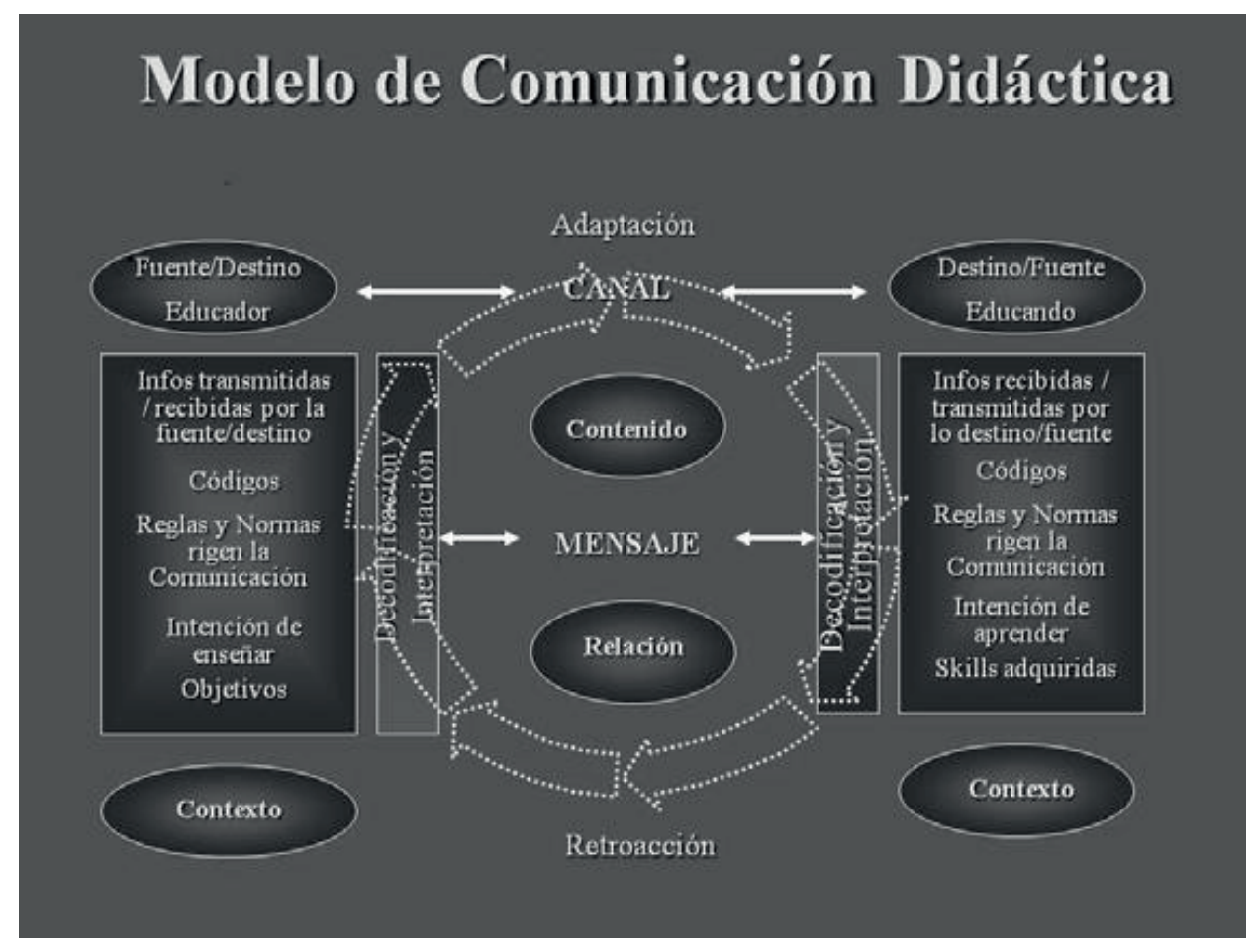

Figura 2. Modelo de comunicación didáctica

Fuente: elaboración propia 
Sin embargo, debido a que el modelo incorpora una gran cantidad de variables que involucran a la situación de comunicación, hay un desafío en relación con los medios para medir la competencia en el ámbito de la relación. Por esa razón, una de las directrices que guia nuestro trabajo futuro es encontrar una metodología para la evaluación empírica de la competencia relacional a fin de permitir la construcción de una teoría de la comunicación didáctica más completa y coherente.

\section{RFERENCIAS}

Au, K. \& Mason, J. (1983). Cultural congruence in classroom participation structures: Achieving a balance of rights. Discourse Processes, 6.

Austin, J. (1971). How to Do Things With Words. Oxford: Oxford University Press.

Baringer, D. \& Mccroskey, J. (2000). Immediacy in the classroom: Student immediacy. Communication Education, 49.

Bernstein, B. (1971). Class, Codes and Control: Theoretical Studies Towards Sociology of Language. London: Routledge \& Kegan Paul.

Bryce-Heath, S. (1983). Ways with words. Cambridge: Cambridge University Press.

Canale, M. \& Swain, M. (1980). Theoretical bases of communicative approaches to second language teaching and testing. Applied Linguistics, 1.

Cazden, C., John, V. \& Hymes, D. (1972). Functions of Language in the Classroom. New York: Teachers College.

Cherry-Wilkinson, L.(1982). Communicating in the Classroom. New York: Academic Press.

Chomsky, N. (1965). Aspects of the Theory of Syntax. Cambridge: The MIT Press.

Collier, M. (2000). Understanding cultural identities in intercultural communication: A ten-step inventory. En Intercultural communication: A Reader. Belmont: Wadsworth.

Crawford, T., Castanheira, M. Dixon, C. \& Green, J. (2001). What counts as literacy: An Interactional ethnographic perspective. En Literacy and the Curriculum: Success in Senior Secondary Schooling. Australia: The Australian Council for Educational Research Ltd..

Cole, P. \& Morgan, J. (1975). Syntax and Semantics (vol. 3). New York: Academic Press. 
Craig, R. (1999). Communication Theory As a Field. Communication Theory, 2(9).

Cumming, J. \& Wyatt-Smith, C. (2001). Literacy and the Curriculum: Success in Senior Secondary Schooling. Australia: The Australian Council for Educational Research Ltd.

Cupach, W.R. \& Imahori, T.T. (1993). Identity management theory: Communication competence in intercultural episodes and relationships. En Intercultural communication competence. Sage: Newbury Park.

Dance, F. E. X. \& Larson, C. E. (1976). The Functions of Human Communication: A Theoretical Approach. New York: Holt, Rinehart \& Winston.

Delamont, S. \& Hamilton, D. (1976). Classroom research: A critique and a new approach. En Explorations in classroom observation. London: John Wiley.

Dervin, B. (1989). Progress in communication sciences (vol. 10) Norwood: Ablex.

Edwards, D., \& Mercer, N. (1987). Common knowledge: The development of understanding in the classroom. New York: Methuen.

Emihovich, C. (1989). Locating learning: Ethnographic perspectives on classroom research. Norwood: Ablex Publishing.

Flanders, N.A. (1970). Analyzing Teaching Behaviour. Addison-Wesley: Reading.

Giles, H., Mulac, A, Bradac, J. \& Johnson, P. (1987). Speech accommodation theory: the first decade and beyond. En Communication yearbook, 10. Sage: Newbury Park.

Grice, H. P. (1975). Logic and Conversation. En Syntax and Semantics (vol. 3). New York: Academic Press.

Griffin, E. (1991). Communication Theory: A First Look. New York: McGraw-Hill.

Gumperz, J. \& Cook-Gumperz, J. (1982). Communicative Competence. En Communicating in the Classroom. New York: Academic Press.

Gumperz, J. e Hymes, D. (1972) Directions in Sociolinguistics: Ethnography of Communication. New York: Holt, Rinehart and Winston.

Gumperz, J. (1984). Communicative Competence Revisited. En Meaning, Form and Use in Context: Linguistic Applications. Washington: Georgetown University Press.

Gumperz, J. (1981). The Linguistic Bases of Communicative Competence. En Analuzing Discourse Text and Talk. Washington: Georgetown Universitv Press. 
Holliday, A. (1999). Small culture. Applied Linguistics, 20(2).

Hymes, D. (1972a). Models of the interaction of language and social life. En Directions in Sociolinguistics: Ethnography of Communication. New York: Holt, Rinehart and Winston.

Hymes, D. (1972b). On communicative competence. En Sociolinguistics. Harmondsworth: Penguin Books.

Imahori, T. \& Lanigan, M. (1989). Relational model of intercultural communication competence. International Journal of Intercultural Relations, 13.

Levinson, S. (1983). Pragmatics. Cambridge: Cambridge University Press.

Littlejohn, S. (1996). Theories of Human Communication. Belmont: Wadsworth Publishing Co.

Mclaughlin, M. (1987). Communication yearbook, 10. Sage: Newbury Park.

Mehan, H. (1985). The structure of classroom discourse. En Handbook of Discourse Analysis. New York:Academic Press.

Mehan, H. (1979). Learning lessons. Cambridge: Harvard University Press.

Nussbaum, J. F. (1992). Effective teacher behaviors. Communication Education, 41.

Pedro, E. (1982). O Discurso na Aula - Uma Análise Sociolinguística da Prática Escolar em Portugal. Lisboa: Edições Rolim.

Pride, J. \& Holmes, J. (1972). Sociolinguistics. Harmondsworth: Penguin Books.

Samovar, L. \& Porter, R. (2000). Intercultural communication: A Reader. Wadsworth. Belmont.

Savignon, S. (1983). Communicative competence: theory and classroom practice; texts and contexts in second language learning. Addison-Wesley: Reading.

Schiffrin, D. (1984). Meaning, Form and Use in Context: Linguistic Applications, G.U.R.T. (Georgetown University Round Table). Washington: Georgetown University Press.

Searle, J. (1970). Speech Acts: An Essay in the Philosophy of Language. London: Cambridge University Press.

Spitzberg. B. \& Hecht. M. (1984). A component model of relational competence. 
Stubbs, M. \& Delamont, S. (1976). Explorations in classroom observation. London: John Wiley.

Tanner, D. (1981). Analyzing Discourse Text and Talk. Washington: Georgetown University Press.

Van Dijk, T. (1985). Handbook of Discourse Analysis (Vol. 3). New York: Academic Press.

Van Ek, J. (1975). The threshold level. Strasbourg: Council of Europe.

Waldeck, J., Kearney, P., \& Plax, T. (2001). Instructional and developmental communication theory and research in the 1990s: Extending the agenda for the 21st century. Communication Yearbook, 24.

Watzlawick, P., Beavin, J. \& Jackson, D. (2000). Pragmática da Comunicação Humana: um estudo dos padrões, patologias e paradoxos da interação (14 ${ }^{\mathrm{a}}$ ed.). São Paulo: Editora Cultrix.

Wiemann, J. (1977). Explication and test of a model of communicative competence. Review of Educational Research, 50.

Wiemann, J. \& Bradac, J. (1989). Metatheoretical issues in the study of communicative competence. En Progress in communication science. Norwood: Ablex.

Wiemann, J. \& Kelly, C. (1981). Pragmatics of interpersonal competence. En Rigor and imagination: Essays from the legacy of Gregory Bateson. New York: Praeger.

Wilder-Mott, C. \& Weakland, J. (1981). Rigor and imagination: Essays from the legacy of Gregory Bateson. New York: Praeger.

Wiseman, R. \& Koester, J. (1993). Intercultural communication competence. Sage: Newbury Park.

Yamauchi, L., \& Tharp, R. (1995). Culturally compatible conversations in Native American classrooms. Linguistics and Education, 7. 


\section{José Manuel Cardoso-Belo}

Doctor en Filosofía y Ciencias de la Educación de la Universidad de Santiago de Compostela y Profesor Asociado con Agregación en el Departamento de Letras, Artes y Comunicación de la Escuela de Ciencias Humanas y Sociales (ECHS) de la Universidad de Trás-os-Montes e Alto Douro (UTAD) en Portugal. Después de haber actuado como Director de Departamento y Director del $2^{\circ}$ Ciclo de estudios (Master) de Ciencias de la Comunicación de la UTAD, es, actualmente, miembro del Consejo Científico de la ECHS y Director del Centro de Estudios en Letras (CEL) donde se desarrollan proyectos de investigación en las áreas de Lingüística, Comunicación, Cultura y Literatura patrocinados y financiados por la Fundación para la Ciencia y la Tecnología (FCT). 